\title{
Rapid development of cross-clade neutralizing antibody responses after clade B gp120/ gp140 protein priming and clade $\mathrm{C}$ gp140 protein boosting
}

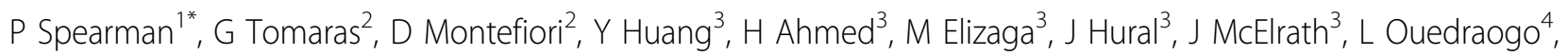
M Pensiero ${ }^{4}$, C Butler $^{4}$, S Kalams ${ }^{5}$, ET Overton', S Barnett ${ }^{7}$, N Group $^{1}$

From AIDS Vaccine 2012

Boston, MA, USA. 9-12 September 2012

\section{Background}

Immunization with heterologous Env protein immunogens following an immunologic rest period has the potential to generate cross-clade neutralizing antibody responses. We identified individuals who had received a clade B Env protein with MF59 4-17 years earlier, most in combination with a DNA or ALVAC prime, and administered a clade $\mathrm{C}$ protein boost in an open label phase 1 trial.

\section{Methods}

Sixteen previously primed volunteers and 20 naïve volunteers each received 2 doses of a clade C TV1 trimeric Env protein with MF59 given 6 months apart. HIV-1 specific CD4+ and CD8+ T cell responses were measured by an intracellular cytokine staining (ICS) assay. Antibody responses were measured with a Luminex binding antibody assay and a neutralizing antibody assay in TZM-bl Cells.

\section{Results}

Despite the long interval, $31 \%$ of primed participants demonstrated $\mathrm{CD} 4+\mathrm{T}$ cell responses to Env at baseline, which increased to $75 \%$ after a single protein boost. IgG and IgA responses to TV1 trimeric Env were present in $64 \%$ (IgG) and 7\% (IgA) of primed participants at baseline, and rose to $93 \%$ and $85 \%$, respectively, after one dose of protein. $71 \%$ of primed participants demonstrated neutralizing antibodies against Tier 1 clade B

${ }^{1}$ Emory University, Atlanta, GA, USA

Full list of author information is available at the end of the article isolate $\mathrm{MN}$ at baseline. After a single booster dose of protein, $100 \%$ of the primed participants neutralized $\mathrm{MN}$ and $93 \%$ showed neutralizing activity against a clade C isolate, MW965.26. Unprimed participants did not demonstrate $\mathrm{CD} 4+$ responses or antibody responses to Env until after the second dose, which elicited IgG and IgA responses to TV1 trimeric Env in $88 \%$ and $50 \%$, respectively. Neutralizing antibody developed to $\mathrm{MN}$ in 38\% and to MW965.26 in $88 \%$ of the unprimed participants.

\section{Conclusion}

These results demonstrate the durability of vaccine-elicited HIV-1 specific antibody responses and support current efforts to enhance the breadth and magnitude of neutralizing antibodies through heterologous protein prime-boost regimens.

Author details

'Emory University, Atlanta, GA, USA. ${ }^{2}$ Department of Surgery, Duke Human Vaccine Institute, Durham, NC, USA. ${ }^{3}$ Fred Hutchinson Cancer Research Center, Seattle, WA, USA. ${ }^{4}$ Division of AIDS, NIAID, NIH, Bethesda, MD, USA. ${ }^{5}$ Vanderbilt University School of Medicine, Nashville, TN, USA. ${ }^{6}$ University of Alabama at Birmingham, Birmingham, AL, USA. ${ }^{7}$ Novartis Vaccines and Diagnostics, Cambridge, MA, USA.

Published: 13 September 2012

doi:10.1186/1742-4690-9-S2-P137

Cite this article as: Spearman et al: Rapid development of cross-clade neutralizing antibody responses after clade B gp120/gp140 protein priming and clade c gp140 protein boosting. Retrovirology 2012 9(Suppl 2):P137. 